\title{
Conservation Laws and Self-Consistent Sources for an Integrable Lattice Hierarchy Associated with a Three-by-Three Discrete Matrix Spectral Problem
}

\author{
Yu-Qing $\mathrm{Li}^{1}$ and Bao-Shu Yin ${ }^{2,3}$ \\ ${ }^{1}$ College of Mathematics and Systems Science, Shandong University of Science and Technology, Qingdao 266590, China \\ ${ }^{2}$ Institute of Oceanology, China Academy of Sciences, Qingdao 266071, China \\ ${ }^{3}$ Key Laboratory of Ocean Circulation and Wave, Chinese Academy of Sciences, Qingdao 266071, China \\ Correspondence should be addressed to Bao-Shu Yin; baoshuyin@126.com
}

Received 25 June 2014; Accepted 14 August 2014; Published 19 October 2014

Academic Editor: Huanhe Dong

Copyright (C) 2014 Y.-Q. Li and B.-S. Yin. This is an open access article distributed under the Creative Commons Attribution License, which permits unrestricted use, distribution, and reproduction in any medium, provided the original work is properly cited.

A lattice hierarchy with self-consistent sources is deduced starting from a three-by-three discrete matrix spectral problem. The Hamiltonian structures are constructed for the resulting hierarchy. Liouville integrability of the resulting equations is demonstrated. Moreover, infinitely many conservation laws of the resulting hierarchy are obtained.

\section{Introduction}

Nonlinear integrable systems of the discrete version, treated as models of some physical phenomena, have attracted more and more attention in recent years. A well-known result is that a hierarchy of soliton equations can be generated through the isospectral compatibility condition of a pair of spectral problems [1]. By using the discrete trace identity the Hamiltonian forms of the soliton equations can be constructed [2]. Various methods have been developed to search for new integrable systems [3-5], integrable coupling systems [6], soliton solutions [7], and so on. However, the work of searching for new integrable systems associated with higher order matrix spectral problems is few. In $[8,9]$, the well-known method is used for the matrix spectral problems with $3 \times 3$ matrixes. In studying the integrability of discrete systems, the conservation laws play important roles. From the Lax pair of lattice soliton equations conservation laws can be deduced directly [10].

With the development of soliton theory, people began to focus on the soliton equations with self-consistent sources. Soliton equations with self-consistent sources are often used to express interactions between different solitary waves and are relevant to some problems of hydrodynamics, solid state physics, plasma physics, and so on. Many integrable coupling systems with self-consistent sources in continuous cases are obtained [11-14]. In [15, 16], integrable discrete systems with self-consistent sources are given.

In the present paper, first, a new three-by-three discrete matrix spectral problem is proposed. By means of constructing a proper continuous time evolution equation and using the discrete zero curvature equation a hierarchy of lattice models is derived. Then the Hamiltonian forms of the resulting hierarchy are worked out by using the discrete trace identity. Further, the Liouville integrability of the discrete systems is demonstrated. Infinitely many conservation laws and self-consistent sources for the integrable systems are also obtained.

\section{A New Three-by-Three Discrete Matrix Spectral Problem and Related Integrable Lattice Hierarchy}

We first recall some presentations on a discrete integrable system. For a lattice function $f_{n}=f(n)$, the shift operator $E$, the inverse of $E$, and the operator $E^{k}$ are defined by

$$
E f_{n}=f(n+1),
$$




$$
\begin{aligned}
E^{-1} f_{n} & =f(n-1), \\
E^{k} f_{n} & =f(n+k), \quad k \in Z .
\end{aligned}
$$

Let $u_{n}=u(n, t)$ be the potential vector. The variational derivative, the Gateaux derivative, the inner product, and the Poisson bracket are defined by

$$
\begin{gathered}
\widetilde{H}_{n}=\sum_{n \in z} H_{n}, \quad \frac{\delta \widetilde{H}_{n}}{\delta u_{n}}=\sum_{m \in z} E^{-m}\left(\frac{\partial H_{n}}{\partial u_{n+m}}\right), \\
P^{\prime}\left(u_{n}\right)\left[v_{n}\right]=\left.\frac{\partial}{\partial \varepsilon} P\left(u_{n}+\varepsilon v_{n}\right)\right|_{\varepsilon=0}, \\
\left\langle f_{n}, g_{n}\right\rangle=\sum_{n \in z}\left(f_{n}, g_{n}\right)_{R^{3}}, \\
\left\{f_{n}, g_{n}\right\}_{J}=\left\langle\frac{\delta f_{n}}{\delta u_{n}}, J \frac{\delta g_{n}}{\delta u_{n}}\right\rangle=\sum_{n \in z}\left(\frac{\delta f_{n}}{\delta u_{n}}, J \frac{\delta g_{n}}{\delta u_{n}}\right)_{R^{3}},
\end{gathered}
$$

where $P$ can be a vector function or an operator, $f_{n}$ and $g_{n}$ are vector functions, $\left(f_{n}, g_{n}\right)_{R^{3}}$ denotes the standard inner product of $f_{n}$ and $g_{n}$ in the Euclidean space $R^{3}$, and $J$ is a Hamiltonian operator. A system of evolution equations $u_{n_{t_{m}}}=K_{m}\left(u_{n}\right)$ is called a Hamiltonian system, if there is a Hamiltonian operator $J$ and a sequence of conserved functionals $\widetilde{H}_{n}^{(m)}, m=1,2, \ldots$, such that

$$
u_{n_{t_{m}}}=K_{m}\left(u_{n}\right)=J \frac{\delta \widetilde{H}_{n}^{(m)}}{\delta u_{n}} .
$$

The functional $\widetilde{H}_{n}^{(m)}$ is called a Hamiltonian functional of the system, and we say that the system possesses Hamiltonian structures. As to a discrete Hamiltonian system, if there are infinitely many involutive conserved functionals, we say it is a Liouville integrable discrete Hamiltonian system.

In this paper, we consider the $3 \times 3$ discrete matrix spectral problem:

$$
E \varphi_{n}=U_{n}\left(u_{n}, \lambda\right) \varphi_{n}, \quad U_{n}\left(u_{n}, \lambda\right)=\left(\begin{array}{ccc}
0 & 1 & 0 \\
0 & 0 & 1 \\
p_{n} & r_{n} & w_{n}+\lambda
\end{array}\right)
$$

in which $u_{n}=\left(p_{n}, r_{n}, w_{n}\right)^{T}$ is the potential, $p_{n}=p(n, t)$, $r_{n}=r(n, t)$, and $w_{n}=w(n, t)$ are real functions defined over $Z \times R, \lambda$ is a spectral parameter, $\lambda_{t}=0$, and $\varphi_{n}=$ $\left(\varphi_{1}(n), \varphi_{2}(n), \varphi_{3}(n)\right)^{T}$ is the eigenfunction.

To get a hierarchy of lattice models associated with (6), first we solve the stationary discrete zero curvature equation:

$$
\left(E \Gamma_{n}\right) U_{n}-U_{n} \Gamma_{n}=0,
$$

where

$$
\Gamma_{n}=\left(\begin{array}{ccc}
a_{n} & b_{n} & c_{n} \\
d_{n} & e_{n} & f_{n} \\
g_{n} & h_{n} & -a_{n}-e_{n}
\end{array}\right) .
$$

Equation (8) gives

$$
\begin{gathered}
p_{n} c_{n+1}=d_{n}, \\
r_{n} c_{n+1}+a_{n+1}=e_{n}, \\
b_{n+1}-f_{n}+c_{n+1}\left(w_{n}+\lambda\right)=0, \\
p_{n} f_{n+1}=g_{n}, \\
r_{n} f_{n+1}+d_{n+1}-h_{n}=0, \\
f_{n+1}\left(w_{n}+\lambda\right)+e_{n+1}+a_{n}+e_{n}=0, \\
-r_{n} d_{n}-\left(w_{n}+\lambda\right) g_{n}=p_{n}\left(a_{n}+a_{n+1}+e_{n+1}\right), \\
-r_{n}\left(a_{n+1}+e_{n+1}+e_{n}\right)+g_{n+1}=p_{n} b_{n}+h_{n}\left(w_{n}+\lambda\right), \\
-r_{n} f_{n}+h_{n+1}=p_{n} c_{n}+\left(a_{n+1}+e_{n+1}-a_{n}-e_{n}\right)\left(w_{n}+\lambda\right) .
\end{gathered}
$$

Substituting the expansions

$$
\begin{array}{ll}
a_{n}=\sum_{m=0}^{\infty} a_{n}^{(m)} \lambda^{-m}, & b_{n}=\sum_{m=0}^{\infty} b_{n}^{(m)} \lambda^{-m}, \\
c_{n}=\sum_{m=0}^{\infty} c_{n}^{(m)} \lambda^{-m}, & d_{n}=\sum_{m=0}^{\infty} d_{n}^{(m)} \lambda^{-m}, \\
e_{n}=\sum_{m=0}^{\infty} e_{n}^{(m)} \lambda^{-m}, & f_{n}=\sum_{m=0}^{\infty} f_{n}^{(m)} \lambda^{-m}, \\
g_{n}=\sum_{m=0}^{\infty} g_{n}^{(m)} \lambda^{-m}, & h_{n}=\sum_{m=0}^{\infty} h_{n}^{(m)} \lambda^{-m}
\end{array}
$$

into (9), we get the recursion relation

$$
\begin{gathered}
p_{n} c_{n+1}^{(m)}=d_{n}^{(m)}, \\
a_{n+1}^{(m)}-e_{n}^{(m)}+r_{n} c_{n+1}^{(m)}=0, \\
b_{n+1}^{(m)}-f_{n}^{(m)}+w_{n} c_{n+1}^{(m)}=-c_{n+1}^{(m+1)}, \\
g_{n}^{(m)}-p_{n} f_{n+1}^{(m)}=0, \\
r_{n} f_{n+1}^{(m)}+d_{n+1}^{(m)}-h_{n}^{(m)}=0, \\
-p_{n}\left(a_{n}^{(m)}+a_{n+1}^{(m)}\right)-w_{n} g_{n}^{(m)}-p_{n} e_{n+1}^{(m)}-r_{n} d_{n}^{(m)}=g_{n}^{(m+1)}, \\
-r_{n}\left(a_{n+1}^{(m)}+e_{n}^{(m)}+e_{n+1}^{(m)}\right)-w_{n} h_{n}^{(m)}+g_{n+1}^{(m)}-p_{n} b_{n}^{(m)}=h_{n}^{(m+1)}, \\
h_{n+1}^{(m)}+w_{n}^{(m)}\left(a_{n}^{(m)}-a_{n+1}^{(m)}+e_{n}^{(m)}-e_{n+1}^{(m)}\right)-p_{n} c_{n}^{(m)}-r_{n} f_{n}^{(m)} \\
=a_{n+1}^{(m+1)}-a_{n}^{(m+1)}+e_{n+1}^{(m+1)}-e_{n}^{(m+1)}
\end{gathered}
$$

and the initial requirement

$$
\begin{gathered}
a_{n+1}^{(0)}+e_{n+1}^{(0)}=a_{n}^{(0)}+e_{n}^{(0)}, \\
g_{n}^{(0)}=h_{n}^{(0)}=d_{n}^{(0)}=f_{n+1}^{(0)}=c_{n+1}^{(0)}=0 .
\end{gathered}
$$


The initial values are taken as $b_{n}^{(0)}=c_{n}^{(0)}=f_{n}^{(0)}=0$, so we can get $e_{n}^{(0)}=a_{n+1}^{(0)}, a_{n}^{(0)}=e_{n+1}^{(0)}$, and then we take the initial value $a_{n}^{(0)}=e_{n}^{(0)}=-1 / 3$. Note that the definition of the inverse operator of $D=(E-1)$ does not yield any arbitrary constant in computing $a_{n}^{(m)}$ and $e_{n}^{(m)}, m \geq 1$. Thus, the recursion relation (11) uniquely determines $a_{n}^{(m)}, b_{n}^{(m)}, c_{n}^{(m)}, d_{n}^{(m)}, e_{n}^{(m)}$, $f_{n}^{(m)}, g_{n}^{(m)}$, and $h_{n}^{(m)}, m \geq 1$, and the first few quantities are given by

$$
\begin{gathered}
a_{n}^{(1)}=b_{n}^{(1)}=c_{n}^{(1)}=d_{n}^{(1)}=e_{n}^{(1)}=0, \\
f_{n}^{(1)}=1, \quad g_{n}^{(1)}=p_{n}, \quad h_{n}^{(1)}=r_{n}, \\
a_{n}^{(2)}=0, \quad b_{n}^{(2)}=1, \quad c_{n}^{(2)}=1, \\
d_{n}^{(2)}=p_{n}, \quad e_{n}^{(2)}=r_{n}, \quad f_{n}^{(2)}=-w_{n}, \\
g_{n}^{(2)}=-p_{n} w_{n}, \quad h_{n}^{(2)}=p_{n+1}-w_{n} r_{n}, \ldots
\end{gathered}
$$

Let

$$
=\left(\begin{array}{lll}
\sum_{i=0}^{m} a_{n}^{(i)} \lambda^{m-i} & \sum_{i=0}^{m} b_{n}^{(i)} \lambda^{m-i} & \sum_{i=0}^{m} c_{n}^{(i)} \lambda^{m-i} \\
\sum_{i=0}^{m} d_{n}^{(i)} \lambda^{m-i} & \sum_{i=0}^{m} e_{n}^{(i)} \lambda^{m-i} & \sum_{i=0}^{m} f_{n}^{(i)} \lambda^{m-i} \\
\sum_{i=0}^{m} g_{n}^{(i)} \lambda^{m-i} & \sum_{i=0}^{m} h_{n}^{(i)} \lambda^{m-i} & -\sum_{i=0}^{m} a_{n}^{(i)} \lambda^{m-i}-\sum_{i=0}^{m} e_{n}^{(i)} \lambda^{m-i}
\end{array}\right),
$$

and then take a modification

$$
\eta_{n}^{(m)}=\left(\begin{array}{ccc}
f_{n-1}^{(m+1)} & c_{n}^{(m+1)} & 0 \\
0 & f_{n}^{(m+1)} & 0 \\
0 & 0 & 0
\end{array}\right), \quad m \geq 0
$$

Now we set

$$
V_{n}^{[m]}=V_{n}^{(m)}+\eta_{n}^{(m)}, \quad m \geq 0 .
$$

Then we introduce the auxiliary spectral problems associated with the spectral problem (6):

$$
\varphi_{n_{t_{m}}}=V_{n}^{[m]} \varphi_{n}, \quad m \geq 0 .
$$

The compatibility conditions of (6) and (17) are

$$
U_{n_{t_{m}}}=\left(E V_{n}^{[m]}\right) U_{n}-U_{n} V_{n}^{[m]}, \quad m \geq 0,
$$

which give rise to the following hierarchy of integrable lattice equations:

$$
\begin{gathered}
p_{n_{t m}}=g_{n}^{(m+1)}-p_{n} f_{n-1}^{(m+1)}, \\
r_{n_{t_{m}}}=h_{n}^{(m+1)}-r_{n} f_{n}^{(m+1)}-p_{n} c_{n}^{(m+1)}, \\
w_{n_{t_{m}}}=\left(a_{n+1}^{(m+1)}-a_{n}^{(m+1)}\right)+\left(e_{n+1}^{(m+1)}-e_{n}^{(m+1)}\right),
\end{gathered}
$$

$$
(m \geq 0) \text {. }
$$

So the discrete spectral problem (6) and (17) constitute the Lax pairs of (19), and (19) are a hierarchy of Lax integrable lattice equations. It is easy to verify that the first lattice equation in (19), when $m=1$, under $t_{1} \rightarrow t$, is

$$
\begin{aligned}
p_{n_{t}} & =p_{n}\left(w_{n-2}-w_{n}\right), \\
r_{n_{t}} & =r_{n}\left(w_{n-1}-w_{n}\right)+\left(p_{n+1}-p_{n}\right), \\
w_{n_{t}} & =r_{n+1}-r_{n} .
\end{aligned}
$$

The Lax pair of (20) is (6) and the time evolution law for $\varphi_{n}$ is as follows:

$$
\begin{aligned}
\varphi_{n_{t}} & =V_{n}^{[1]} \varphi_{n} \\
& =\left(\begin{array}{ccc}
-\frac{\lambda}{3}-w_{n-2}+\frac{2}{3} & 1 & 0 \\
0 & -\frac{\lambda}{3}+\frac{2}{3}-w_{n-1} & 1 \\
p_{n} & r_{n} & \frac{2}{3} \lambda+\frac{2}{3}
\end{array}\right) \varphi_{n} .
\end{aligned}
$$

Now we would like to derive the Hamiltonian structures for (19).

Set $R_{n}=\Gamma_{n} U_{n}^{-1}$; through a direct calculation, we get

$$
\begin{array}{ll}
\operatorname{Tr}\left(R_{n} \frac{\partial U_{n}}{\partial \lambda}\right)=\frac{g_{n}}{p_{n}}, & \operatorname{Tr}\left(R_{n} \frac{\partial U_{n}}{\partial p_{n}}\right)=\frac{a_{n}}{p_{n}}, \\
\operatorname{Tr}\left(R_{n} \frac{\partial U_{n}}{\partial r_{n}}\right)=\frac{d_{n}}{p_{n}}, & \operatorname{Tr}\left(R_{n} \frac{\partial U_{n}}{\partial w_{n}}\right)=\frac{g_{n}}{p_{n}} .
\end{array}
$$

By the discrete trace identity [2]

$$
\begin{array}{r}
\frac{\delta}{\delta u_{n}^{i}} \sum_{n \in Z} \operatorname{Tr}\left(R_{n} \frac{\partial U_{n}}{\partial \lambda}\right)=\lambda^{-\varepsilon}\left(\frac{\partial}{\partial \lambda}\right) \lambda^{\varepsilon} \operatorname{Tr}\left(R_{n} \frac{\partial U_{n}}{\partial u_{n}^{i}}\right), \\
i=1,2,3,
\end{array}
$$

we have

$$
\left(\frac{\delta}{\delta p_{n}}, \frac{\delta}{\delta r_{n}}, \frac{\delta}{\delta w_{n}}\right)^{T} \sum_{n \in Z}\left(\frac{g_{n}}{p_{n}}\right)=\lambda^{-\varepsilon}\left(\frac{\partial}{\partial \lambda}\right) \lambda^{\varepsilon}\left(\begin{array}{c}
\frac{a_{n}}{p_{n}} \\
\frac{d_{n}}{p_{n}} \\
\frac{g_{n}}{p_{n}}
\end{array}\right),
$$

where $\varepsilon$ is a constant to be found. 
By substituting

$$
\begin{aligned}
& a_{n}=\sum_{m=0}^{\infty} a_{n}^{(m)} \lambda^{-m}, \\
& d_{n}=\sum_{m=0}^{\infty} d_{n}^{(m)} \lambda^{-m}, \\
& g_{n}=\sum_{m=0}^{\infty} g_{n}^{(m)} \lambda^{-m}
\end{aligned}
$$

into (24) and equating the coefficients of $\lambda^{-m-1}$, we have

$$
\left(\frac{\delta}{\delta p_{n}}, \frac{\delta}{\delta r_{n}}, \frac{\delta}{\delta w_{n}}\right)^{T} \sum_{n \in Z}\left(\frac{g_{n}^{(m+1)}}{-m p_{n}}\right)=\left(\begin{array}{c}
\frac{a_{n}^{(m)}}{p_{n}} \\
\frac{d_{n}^{(m)}}{p_{n}} \\
\frac{g_{n}^{(m)}}{p_{n}}
\end{array}\right), \quad m>0 .
$$

Now we can rewrite (19) as follows:

$$
\left(\begin{array}{c}
p_{n} \\
r_{n} \\
w_{n}
\end{array}\right)_{t_{m}}=J\left(\begin{array}{c}
\frac{a_{n}^{(m)}}{p_{n}} \\
\frac{d_{n}^{(m)}}{p_{n}} \\
\frac{g_{n}^{(m)}}{p_{n}}
\end{array}\right)
$$

where

$$
\begin{aligned}
J & =\left(\begin{array}{lll}
J_{11} & J_{12} & J_{13} \\
J_{21} & J_{22} & J_{23} \\
J_{31} & J_{32} & J_{33}
\end{array}\right), \\
J_{11} & =p_{n}\left(E^{-1}-E+E^{-2}-E^{2}\right) p_{n}, \\
J_{12} & =p_{n}\left(E^{-2}+E^{-1}-E-1\right) r_{n}, \\
J_{13} & =p_{n}\left(E^{-2}-1\right) w_{n}, \\
J_{21} & =r_{n}\left(E^{-1}+1-E-E^{2}\right) p_{n}, \\
J_{22} & =r_{n}\left(E^{-1}-E\right) r_{n}+p_{n} E^{-1} w_{n}-w_{n} E p_{n}, \\
J_{23} & =E p_{n}-p_{n} E^{-2}-r_{n}\left(1-E^{-1}\right) w_{n}, \\
J_{31} & =w_{n}\left(1-E^{2}\right) p_{n}, \\
J_{32} & =E^{2} p_{n}-p_{n} E^{-1}+w_{n}(1-E) r_{n}, \\
J_{33} & =E r_{n}-r_{n} E^{-1} .
\end{aligned}
$$

It is easy to verify that the operator $J$ is a Hamiltonian operator. So the lattice systems (19) can be rewritten as the hierarchy of discrete Hamiltonian equation (27). Set

$$
\begin{gathered}
\widetilde{H}_{n}^{(m)}=\sum_{n \in Z} \frac{g_{n}^{(m+1)}}{-m p_{n}}, \quad m \geq 1, \\
\frac{\delta \widetilde{H}_{n}^{(m)}}{\delta u_{n}}=\phi_{n} \frac{\delta \widetilde{H}_{n}^{(m-1)}}{\delta u_{n}} .
\end{gathered}
$$

From the recursion relation (11) we can get the recursion operator $\phi_{n}$ in (30):

$$
\begin{gathered}
\phi_{n}=\left(\begin{array}{ccc}
\phi_{11} & \phi_{12} & \phi_{13} \\
\phi_{21} & \phi_{22} & \phi_{23} \\
\phi_{31} & \phi_{32} & \phi_{33}
\end{array}\right), \\
\phi_{11}=\frac{1}{p_{n}}\left(E^{2}-1\right)^{-1} w_{n}\left(1-E^{2}\right) p_{n} \\
+\frac{1}{p_{n}}(E+1)^{-1} r_{n}\left(E p_{n}+p_{n} E^{-1}\right)^{-1} r_{n}(E-1) p_{n}, \\
\phi_{12}=\frac{1}{p_{n}}\left(E^{2}-1\right)^{-1}\left[E^{2} p_{n}-p_{n} E^{-1}+w_{n}(1-E) r_{n}\right] \\
-\frac{1}{p_{n}}(E+1)^{-1} r_{n}\left(E p_{n}+p_{n} E^{-1}\right)^{-1} \\
\times\left(p_{n} E^{-1} w_{n}-w_{n} E p_{n}\right), \\
\phi_{13}=\frac{1}{p_{n}}\left(E^{2}-1\right)^{-1}\left(E r_{n}-r_{n} E^{-1}\right) \\
\phi_{22}=\left(E p_{n}+p_{n} E^{-1}\right)^{-1}\left(p_{n} E^{-1} w_{n}-r_{n} E p_{n}\right), \\
\phi_{23}=\left(E p_{n}+p_{n} E^{-1}\right)^{-1}\left(E p_{n}-p_{n} E^{-2}\right), \\
\phi_{21}=-\left(E p_{n}+p_{n} p_{n} r_{n}\left(E p_{n}+p_{n} E^{-1}\right)^{-1}\left(E p_{n}-p_{n} E^{-2}\right),\right. \\
\phi_{n} p_{n}, \\
r_{n}(E-1) p_{n},
\end{gathered}
$$

Therefore, we have

$$
M=J \phi_{n}=\left(\begin{array}{ccc}
0 & 0 & p_{n}\left(1-E^{-2}\right) \\
0 & E p_{n}-p_{n} E^{-1} & r_{n}\left(1-E^{-1}\right) \\
\left(E^{2}-1\right) p_{n} & (E-1) r_{n} & 0
\end{array}\right) .
$$


It is easy to verify that $M^{*}=-M$; moreover, we can prove that

$$
\begin{aligned}
& \left\{\widetilde{H}_{n}^{(m)}, \widetilde{H}_{n}^{(l)}\right\}_{J}=0, \quad m, l \geq 1, \\
\left(\widetilde{H}_{n}^{(m)}\right)_{t_{l}}= & \left\langle\frac{\delta \widetilde{H}_{n}^{(m)}}{\delta u_{n}}, u_{n t_{l}}\right\rangle=\left\langle\frac{\delta \widetilde{H}_{n}^{(m)}}{\delta u_{n}}, J \frac{\delta \widetilde{H}_{n}^{(l)}}{\delta u_{n}}\right\rangle \\
= & \left\{\widetilde{H}_{n}^{(m)}, \widetilde{H}_{n}^{(l)}\right\}_{J}=0, \quad m, l \geq 1 .
\end{aligned}
$$

So we get the following.

Proposition 1. $\left\{\widetilde{H}_{n}^{(m)}\right\}_{m \geq 1}$ defined by (29) forms an infinite set of conserved functionals of the hierarchy (19), and $\widetilde{H}_{n}^{(m)}, m \geq 1$, are involution in pairs with respect to the Poisson bracket (4).

Theorem 2. The lattice equations in (19) are all discrete Liouville integrable Hamiltonian systems.

\section{Infinitely Many Conservation Laws}

We can get the following alternative form from (6) and (21):

$$
\begin{gathered}
\varphi_{3}(n+1)=p_{n} \varphi_{3}(n-2)+r_{n} \varphi_{3}(n-1)+\left(w_{n}+\lambda\right) \varphi_{3}(n) \\
\varphi_{3_{t}}(n)=p_{n} \varphi_{3}(n-2)+r_{n} \varphi_{3}(n-1)+\frac{2}{3}(1+\lambda) \varphi_{3}(n) .
\end{gathered}
$$

Set

$$
\theta_{n}=\theta(n)=\frac{\varphi_{3}(n)}{\varphi_{3}(n+1)},
$$

and we can obtain

$$
-\left(\ln \theta_{n}\right)_{t}=(E-1)\left[p_{n} \theta_{n-2} \theta_{n-1}+r_{n} \theta_{n-1}+\frac{2}{3}(1+\lambda)\right] \text {. }
$$

Equation (34) can be written as follows:

$$
\lambda \theta_{n}=1-p_{n} \theta_{n-2} \theta_{n-1} \theta_{n}-r_{n} \theta_{n-1} \theta_{n}-w_{n} \theta_{n} .
$$

Then, expanding $\theta_{n}$ in the power series of $1 / \lambda$ :

$$
\theta_{n}=\sum_{j=1}^{\infty} \theta_{n}^{(j)} \lambda^{-j}
$$

and substituting it into (38), we can obtain all the coefficients $\theta_{n}^{(j)}$. Substitute them into (37) and due to $\theta_{n}^{(1)}=1$ and $\lambda_{t}=0$, we can get the following fact:

$$
\begin{aligned}
& {\left[\sum_{k=1}^{\infty}(-1)^{k} \frac{1}{k}\left(\sum_{j=1}^{\infty} \frac{\theta_{n}^{(j+1)}}{\lambda^{j}}\right)^{k}\right]_{t}} \\
& =(E-1) \\
& \quad \times\left[p_{n} \sum_{j=1}^{\infty} \frac{\theta_{n-2}^{(j)}}{\lambda^{j}} \sum_{j=1}^{\infty} \frac{\theta_{n-1}^{(j)}}{\lambda^{j}}+r_{n} \sum_{j=1}^{\infty} \frac{\theta_{n-1}^{(j)}}{\lambda^{j}}+\frac{2}{3}(1+\lambda)\right],
\end{aligned}
$$

from which an infinite number of conservation laws can be determined by equating the powers of $1 / \lambda$. The following are the first three of them:

$$
\begin{gathered}
w_{n_{t}}=(E-1) r_{n} \\
\left(-r_{n}-\frac{1}{2} w_{n}^{2}\right)_{t}=(E-1)\left(-w_{n-1} r_{n}+p_{n}\right),
\end{gathered}
$$

$$
\begin{aligned}
& \left(p_{n}-r_{n} w_{n-1}-w_{n} r_{n}+\frac{1}{3} w_{n}^{3}\right)_{t} \\
& \quad=(E-1)\left[r_{n}\left(r_{n-1}+w_{n-1}^{2}\right)-p_{n}\left(w_{n-1}+w_{n-2}\right)\right] .
\end{aligned}
$$

We can get other conservation laws in the hierarchy (19) similarly.

\section{Self-Consistent Sources for the Lattice Hierarchy (19)}

In this section, we will construct the lattice hierarchy (19) with self-consistent sources. Consider the auxiliary linear problems

$$
\begin{aligned}
& E\left(\begin{array}{c}
\varphi_{1 j} \\
\varphi_{2 j} \\
\varphi_{3 j}
\end{array}\right)=U_{n}\left(u_{n}, \lambda_{j}\right)\left(\begin{array}{c}
\varphi_{1 j} \\
\varphi_{2 j} \\
\varphi_{3 j}
\end{array}\right), \\
& \left(\begin{array}{c}
\varphi_{1 j} \\
\varphi_{2 j} \\
\varphi_{3 j}
\end{array}\right)_{t_{m}}=V_{n}^{[m]}\left(u_{n}, \lambda_{j}\right)\left(\begin{array}{c}
\varphi_{1 j} \\
\varphi_{2 j} \\
\varphi_{3 j}
\end{array}\right),
\end{aligned}
$$

and, based on the results in [17], we show the following equation:

$$
\frac{\delta \widetilde{H}_{n}^{(m)}}{\delta u_{n}}+\sum_{j=1}^{N} \frac{\delta \lambda_{j}}{\delta u_{n}}=0
$$

where

$$
\begin{gathered}
\frac{\delta \lambda_{j}}{\delta u_{n}}=\frac{1}{2} \operatorname{Tr}\left(\psi_{j} \frac{\partial U\left(u_{n}, \lambda_{j}\right)}{\partial u_{n}}\right), \\
\psi_{j}=\left(\begin{array}{lll}
\varphi_{1 j} \varphi_{1 j} & \varphi_{1 j} \varphi_{2 j} & \varphi_{1 j} \varphi_{3 j} \\
\varphi_{2 j} \varphi_{1 j} & \varphi_{2 j} \varphi_{2 j} & \varphi_{2 j} \varphi_{3 j} \\
\varphi_{3 j} \varphi_{1 j} & \varphi_{3 j} \varphi_{2 j} & \varphi_{3 j} \varphi_{3 j}
\end{array}\right), \quad j=1,2, \ldots, N .
\end{gathered}
$$


Through a direct computation, we obtain the lattice hierarchy with self-consistent sources as follows:

$$
\begin{aligned}
\left(\begin{array}{c}
p_{n} \\
r_{n} \\
w_{n}
\end{array}\right)_{t_{m}}=J & \left(\begin{array}{c}
\frac{\delta \widetilde{H}_{n}^{(m)}}{\delta p_{n}}+\sum_{j=1}^{N} \frac{\delta \lambda_{j}}{\delta p_{n}} \\
\frac{\delta \widetilde{H}_{n}^{(m)}}{\delta r_{n}}+\sum_{j=1}^{N} \frac{\delta \lambda_{j}}{\delta r_{n}} \\
\frac{\delta \widetilde{H}_{n}^{(m)}}{\delta w_{n}}+\sum_{j=1}^{N} \frac{\delta \lambda_{j}}{\delta w_{n}}
\end{array}\right) \\
=J & \left(\begin{array}{c}
\frac{\delta \widetilde{H}_{n}^{(m)}}{\delta p_{n}} \\
\frac{\delta \widetilde{H}_{n}^{(m)}}{\delta r_{n}} \\
\frac{\delta \widetilde{H}_{n}^{(m)}}{\delta w_{n}}
\end{array}\right)+J\left(\begin{array}{c}
\frac{1}{2} \sum_{j=1}^{N} \varphi_{1 j} \varphi_{3 j} \\
\frac{1}{2} \sum_{j=1}^{N} \varphi_{2 j} \varphi_{3 j} \\
\frac{1}{2} \sum_{j=1}^{N} \varphi_{3 j} \varphi_{3 j}
\end{array}\right), m \geq 1 .
\end{aligned}
$$

\section{Conflict of Interests}

The authors declare that there is no conflict of interests regarding the publication of this paper.

\section{Acknowledgments}

This work is supported by the Project Global Change and AirSea Interaction (GASI-03-01-01-02), Nature Science Foundation of Shandong Province of China (no. ZR2013AQ017), Science and Technology Plan Project of Qingdao (no. 142-4-77-jch), Open Fund of the Key Laboratory of Ocean Circulation and Waves, Chinese Academy of Science (no. KLOCAW1401), and Open Fund of the Key Laboratory of Data Analysis and Application, State Oceanic Administration (no. LDAA-2013-04).

\section{References}

[1] G. Z. Tu, "The trace identity, a powerful tool for constructing the Hamiltonian structure of integrable systems," Journal of Mathematical Physics, vol. 30, no. 2, pp. 330-338, 1989.

[2] G. Z. Tu, "A trace identity and its applications to the theory of discrete integrable systems," Journal of Physics A: Mathematical and General, vol. 23, no. 17, pp. 3903-3922, 1990.

[3] W. X. Ma and B. Fuchssteiner, "Integrable theory of the perturbation equations," Chaos, Solitons and Fractals, vol. 7, no. 8, pp. 1227-1250, 1996.

[4] X. Xu and Y. Zhang, "A hierarchy of Lax integrable lattice equations, LIOuville integrability and a new integrable symplectic map," Communications in Theoretical Physics, vol. 41, no. 3, pp. 321-328, 2004.

[5] W. Ma and X. Xu, "A modified Toda spectral problem and its hierarchy of bi-Hamiltonian lattice equations," Journal of Physics A. Mathematical and General, vol. 37, no. 4, pp. 13231336, 2004.
[6] W. Ma, "Enlarging spectral problems to construct integrable couplings of soliton equations," Physics Letters A, vol. 316, no. 1-2, pp. 72-76, 2003.

[7] Y. T. Wu and X. G. Geng, "A new hierarchy of integrable differential-difference equations and Darboux transformation," Journal of Physics A: Mathematical and General, vol. 31, no. 38, pp. 677-684, 1998.

[8] Y. Li, "Two $3 \times 3$ discrete matrix spectral problems and associated Liouville integrable lattice soliton equations," Communications in Nonlinear Science and Numerical Simulation, vol. 16, no. 6, pp. 2493-2500, 2011.

[9] X. G. Geng and D. L. Du, "Two hierarchies of new nonlinear evolution equations associated with matrix spectral problems," Chaos, Solitons and Fractals, vol. 29, pp. 1165-1172, 2006.

[10] D. J. Zhang and D. Y. Chen, "The conservation laws of some discrete soliton systems," Chaos, Solitons \& Fractals, vol. 14, no. 4, pp. 573-579, 2002.

[11] F.-J. Yu and L. Li, "Integrable coupling system of JM equations hierarchy with self-consistent sources," Communications in Theoretical Physics, vol. 53, no. 1, pp. 6-12, 2010.

[12] T. C. Xia, "Two new integrable couplings of the soliton hierarchies with self-consistent sources," Chinese Physics B, vol. 19, no. 10, Article ID 100303, 2010.

[13] H. W. Yang, H. H. Dong, and B. S. Yin, "Nonlinear integrable couplings of a nonlinear Schrödinger-modified Korteweg de Vries hierarchy with self-consistent sources," Chinese Physics B, vol. 21, no. 10, Article ID 100204, 2012.

[14] H. Yang, H. Dong, B. Yin, and Z. Liu, "Nonlinear biintegrable couplings of multicomponent Guo hierarchy with self-consistent sources," Advances in Mathematical Physics, vol. 2012, Article ID 272904, 14 pages, 2012.

[15] F. Yu, "Non-isospectral integrable couplings of Ablowitz-Ladik hierarchy with self-consistent sources," Physics Letters A, vol. 372, no. 46, pp. 6909-6915, 2008.

[16] F. J. Yu and L. Li, "A Blaszak-Marciniak lattice hierarchy with self-consistent sources," International Journal of Modern Physics $B$, vol. 25, no. 25, pp. 3371-3379, 2011.

[17] Y. Zeng, W. Ma, and R. Lin, "Integration of the soliton hierarchy with self-consistent sources," Journal of Mathematical Physics, vol. 41, no. 8, pp. 5453-5489, 2000. 


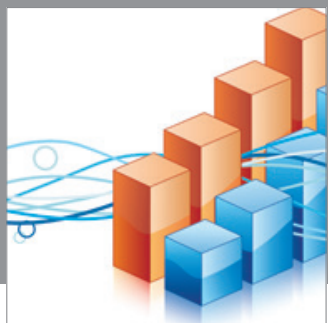

Advances in

Operations Research

mansans

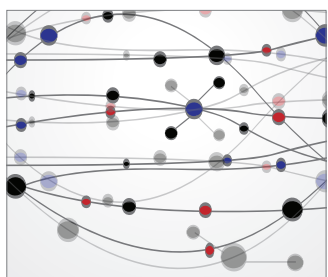

The Scientific World Journal
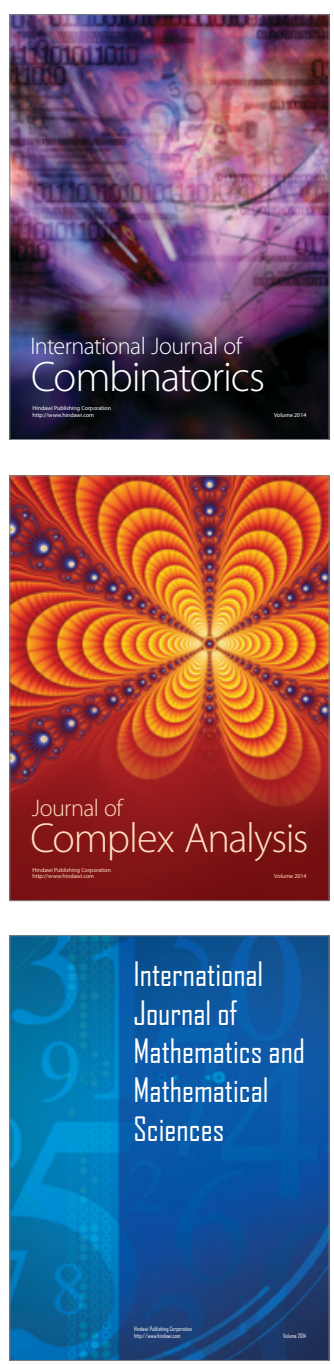
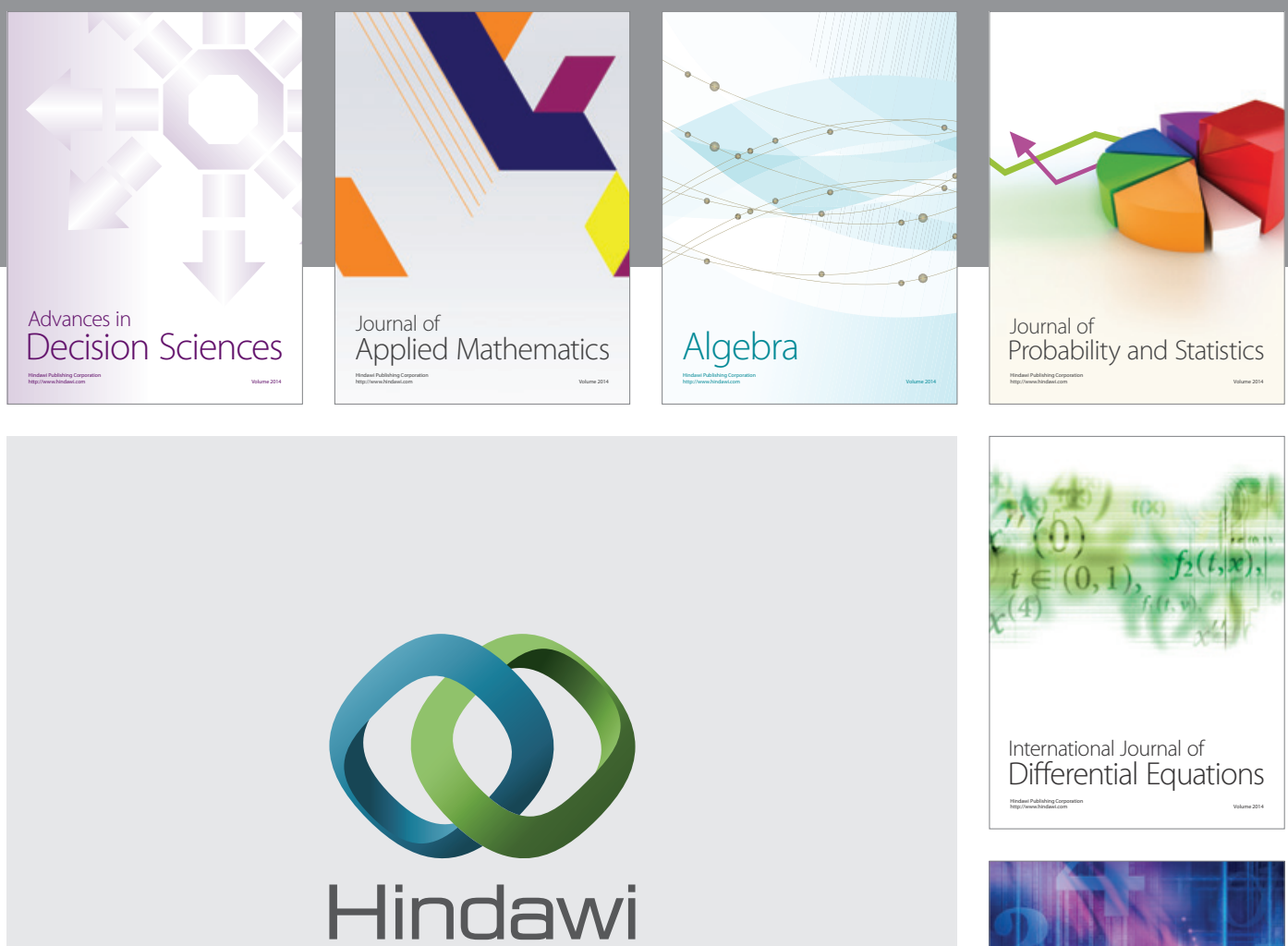

Submit your manuscripts at http://www.hindawi.com
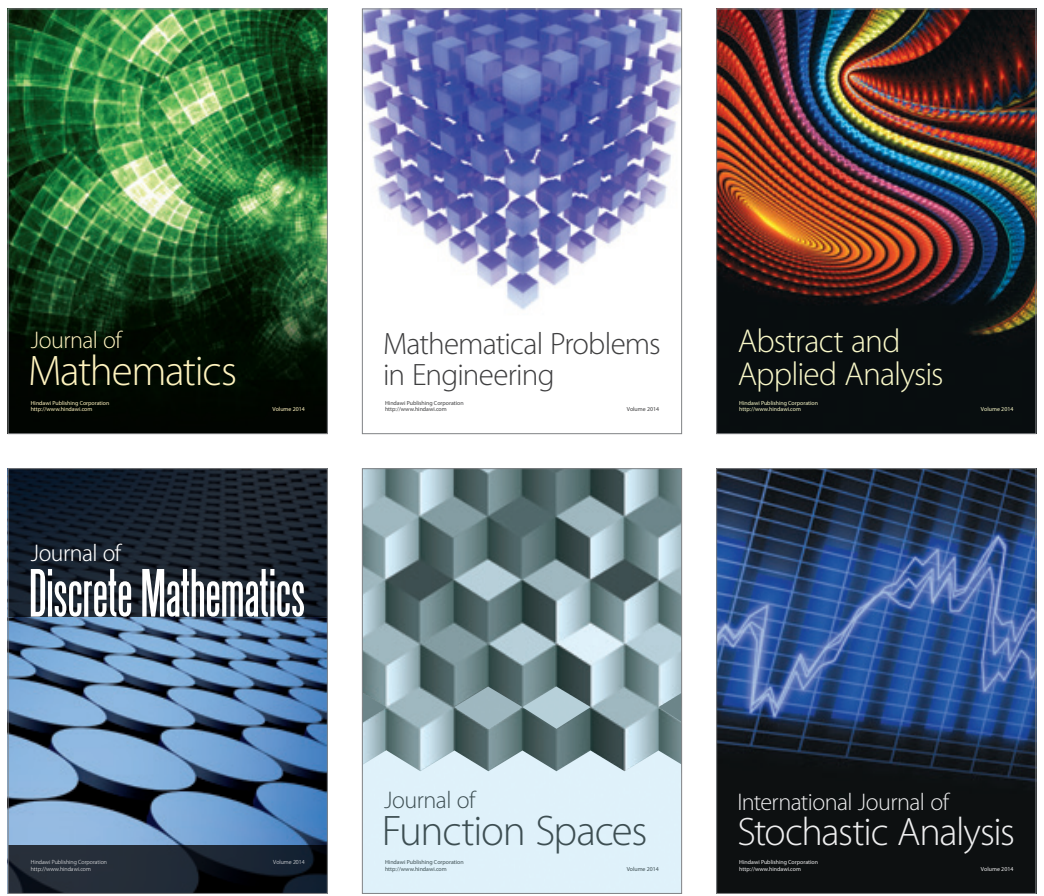

Journal of

Function Spaces

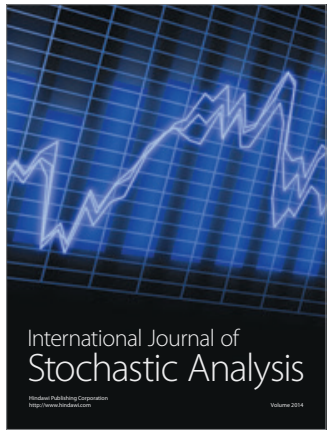

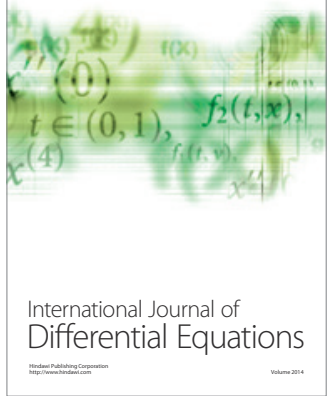
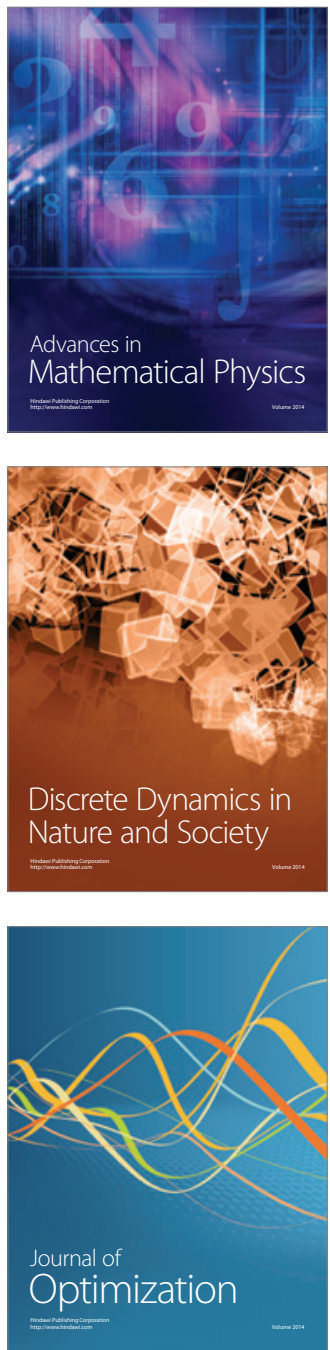